\title{
Development of a mechanical strain amplifying transducer with Bragg grating sensor for low-amplitude strain sensing
}

\author{
Urszula Nawrot ${ }^{1,3}$, Thomas Geernaert ${ }^{1,3}$, Ben De Pauw ${ }^{1,3}$, Dimitrios Anastasopoulos ${ }^{2}$, Edwin Reynders ${ }^{2}$, \\ Guido De Roeck ${ }^{2}$, Francis Berghmans ${ }^{1,3}$ \\ ${ }^{1}$ Vrije Universiteit Brussel, Department of Applied Physics and Photonics, Brussels Photonics (B-PHOT), Pleinlaan 2, B-1050 \\ Brussels, Belgium \\ ${ }^{2}$ University of Leuven (KU Leuven), Department of Civil Engineering, Structural Mechanics Section, Kasteelpark Arenberg \\ 40 - bus 2448, B-3001 Leuven, Belgium \\ ${ }^{3}$ Flanders Make, Oude Diestersebaan 133, B-3920 Lommel, Belgium \\ E-mail: urszula.nawrot@vub.be
}

\begin{abstract}
Vibration-based damage identification is a well-known method to support health monitoring of civil engineering structures. Damage in such structures can be identified by measuring changes of the natural frequencies, damping factors or modal displacements of the structure. However, this approach suffers from the low sensitivity of these natural frequencies and modal displacements to certain types of damage. Modal strains and curvatures can be more sensitive to local damage, but direct monitoring of these quantities with sufficient spatial resolution is not possible with current measurement techniques due to the very small strain levels (submicrostrain) caused by ambient or operational excitation. To deal with this issue, we propose a novel mechanical transducer equipped with an optical fiber Bragg grating (FBG) sensor that enhances the sensitivity to strain with a factor larger than 30 . The principle of operation of the transducer exploits a symmetric cantilever structure that enlarges the strain experienced by the FBG sensor compared to the strain applied to the transducer itself. We carried out dynamic and static tests to verify the ability of the strain-amplifying transducers to measure small-amplitude strain levels and to evidence the potential for carrying out FBG based modal strain measurements on concrete civil engineering structures.
\end{abstract}

\section{Introduction}

Vibration-based damage identification builds on the principle that the modal characteristics of a structure are stiffness dependent. Changes in natural frequencies, damping ratios and modal displacements, which are obtained from successive modal tests, can therefore be used as indicators to detect and to identify damage as the latter impacts structural stiffness. Compared to other approaches for structural damage identification, operational vibration-based damage identification has the advantages of (1) being nondestructive, (2) being able to identify damage that is invisible at the surface, and (3) being 'global' because no a priori knowledge about the location of the damage is required, as opposed to local methods such as ultrasonic testing [1]. Furthermore, this technique can also be applied when only the structural response to ambient (or operational) excitation is measured (so-called operational modal analysis), which means that no artificial excitation by means of pre-defined loads acting on the structure is required. Although vibration-based damage identification is conceptually very attractive, its application to civil structures under ambient excitation faces two major challenges: (1) environmental factors such as temperature also influence the dynamic characteristics and, most importantly, (2) the sensitivity of dynamic characteristics such as natural frequencies, damping ratios and modal displacements to a certain type of damage may be low [2].

In this paper we propose a solution to tackle the latter challenge. The sensitivity to damage can be improved by considering dynamic measurements of modal strains or curvatures instead, as these can be more sensitive to local damage than most other dynamic characteristics $[3,4,5]$. However, the direct 
in-situ dynamic measurement of such strains on a civil engineering structure caused by ambient excitation due to wind or traffic is often problematic because of the very low strain amplitudes, which require a strain sensor with a measurement resolution close to $0.1 \mu \varepsilon$ (microstrain), and preferably better. Strain measurements conventionally rely on the use of resistive strain gauges. These devices are well-known, widely commercially available and standardized. However, resistive strain gauges are also known to feature a limited strain sensitivity $(\sim 1 \mu \varepsilon)$, to suffer from drift, to be sensitive to moisture and to be susceptibility to electromagnetic interference (EMI). An interesting alternative is offered by optical fiber based sensors. These have enjoyed a continuously increasing popularity for civil structural health monitoring because of their immunity to electromagnetic interference, ability to measure over very long distances, and applicability in harsh environments [6]. Optical sensors based on fiber Bragg grating (FBG) technology, in particular, have an important additional advantage as many of such sensors can be wavelength-multiplexed into the same optical fiber, which allows for significant reductions in cabling and installation time [7].

An FBG is formed by a periodic modulation of the refractive index in the core of an optical fiber. When broadband light propagates along the FBG, only a very narrow band of wavelengths is reflected. The central wavelength of this band is commonly called the Bragg wavelength and is given by

$$
\lambda_{B}=2 n_{\mathrm{eff}} \Lambda
$$

where $n_{\text {eff }}$ is the effective refractive index and $\Lambda$ is the grating period [7]. FBG-based sensing of strain or temperature relies on measuring the Bragg wavelength shift given by

$$
\frac{\Delta \lambda_{B}}{\lambda_{B}}=K_{\varepsilon} \Delta \varepsilon+K_{T} \Delta T
$$

where $\mathrm{T}$ is the temperature, $\varepsilon$ is the strain and $K_{\varepsilon}, K_{T}$ are the strain and temperature sensitivity coefficients, respectively. For a FBG fabricated in silica fiber at a central wavelength around $1550 \mathrm{~nm}$, the strain sensitivity is typically $1.2 \mathrm{pm} / \mu \varepsilon$ and the temperature sensitivity is $10.3 \mathrm{pm} /{ }^{\circ} \mathrm{C}$. The strain measurement resolution of FBG sensor based systems is generally restricted to $1 \mu \varepsilon[8,9]$. This can nevertheless be improved to about $0.02 \mu \varepsilon$ by employing passive filter techniques [10], but then each FBG sensor requires a different filter, which complicates the implementation when addressing a dense grid of such sensors.

To enable the measurement of very low strains we propose to combine a FBG sensor with a dedicated mechanical transducer that mechanically amplifies the strain applied to an FBG sensor compared to that applied to the transducer itself. Since the specific target of our research is to use this strain-amplifying transducer to detect and locate damage on concrete bridges (note that $86 \%$ of the bridges in Europe are made of concrete [11]), it should allow not only for static, but also for dynamic strain measurements and it should meet the following set of specifications.

- The average strain should be measured over a sufficient length to allow for integrating the spatial strain variations inherent to the inhomogeneous nature of concrete. On the other hand, the gauge length should not be too large such that multiple transducers can be mounted to enable quasi-distributed measurements over the entire concrete structure.

- The transducer's eigenfrequencies should be sufficiently high to avoid interference with the typical resonance frequencies of civil structures such as long pre-stressed concrete bridges. We consider that the lowest eigenfrequency should exceed $50 \mathrm{~Hz}$.

- The stiffness of the transducer should be negligible when compared to the local stiffness of the civil structure in the region where it is mounted. We consider that the spring constant has to be lower than $10^{7} \mathrm{~N} / \mathrm{m}$. 
- The transducer should be straightforwardly installable and replaceable, without requiring expert knowledge in the handling of optical fibers and should be manufacturable at low cost with high repeatability.

- Finally and most importantly, the transducer should enable strain measurements in both tension and compression, and should result in strain levels experienced by the FBG sensor at least 10 times larger than those experienced by the structure and hence by the transducer itself, in order to provide for the required measurement resolution.

In the remainder of this paper we describe how we addressed this set of requirements. We have structured our manuscript as follows. First, we shortly describe the state-of-the-art in FBG sensor based strain amplifying transducers in Section 2. In Section 3 we introduce the principle of our novel strainamplifying transducer and we analyse the strain sensitivity of the transducer based on a finite element (FE) method. Section 4 details the fabricated transducer and reports on the tensile tests carried out to characterize and calibrate the sensor. We provide the experimental results of static and dynamic testing on a full scale concrete beam in Section 5 and we close our paper with a summary and conclusions in Section 6.

\section{State-of-the-art}

As introduced above, fiber Bragg grating sensors offer a number of clear advantages, but their typical strain measurement resolution is comparable to that of electrical strain gauges [12] in the sense that measuring strain levels below 1 microstrain remains challenging. Several research efforts therefore already aimed to increase the strain resolution of these sensors.

Approaches to do so mostly rely on long-gauge packages that transfer most of the displacement applied to the fixation points of the package to the region holding the FBG, which is significantly shorter than the distance between the fixation points and hence results in a larger strain level when compared to the relative elongation of the distance between the fixation points. Other techniques rely either on a reduced cross-section of the package or on a package composed of materials with different Young moduli, or a combination of both. For these techniques, the absolute change of the sensor gauge length due to structural deformation is imposed largely on that part featuring the lower stiffness. Table 1 summarizes examples reported in the literature with the achieved strain amplification $A$, where $A$ is defined as

$$
A=\frac{\varepsilon_{f}}{\varepsilon_{T}}=\frac{\Delta L_{f} / L_{f}}{\Delta L_{T} / L_{T}}
$$

and $L_{f}$ is the gauge length over which the FBG sensor measures the amplified strain, $\Delta L_{f}$ is the change of this length under applied load, $L_{T}$ is the length of the transducer corresponding to the distance between the two locations at which the load is actually applied. $\Delta L_{T}$ is the change of this transducer length due to the applied load (see for example figure 1). 
Table 1. Summary of mechanical strain amplification of FBG sensors.

\begin{tabular}{cccc}
\hline No. & Amplification Technique & $\begin{array}{c}\text { Strain } \\
\text { amplification (A) }\end{array}$ & Ref. \\
\hline 1 & reduced cross-section of the package & 1.63 & {$[13]$} \\
2 & different Young modulus of materials & 4.40 & {$[14]$} \\
3 & reduced cross-section of the package & 2.30 & {$[15]$} \\
4 & reduced cross-section of the package & 1.33 & {$[16]$} \\
\hline
\end{tabular}

Strain amplification by means of the approaches summarized in table 1 fails to fulfill several of the specifications outlined in Section 1. We illustrate this with two examples below.

A transducer design (figure 1) with the same outer dimensions as presented in this paper (380 x $105 \mathrm{~mm})$, but based on a reduced cross-section [13] can feature a strain amplification of 10 . However, it suffers from a weak central connection that is susceptible to undesirable in-plane and outof-plane bending.

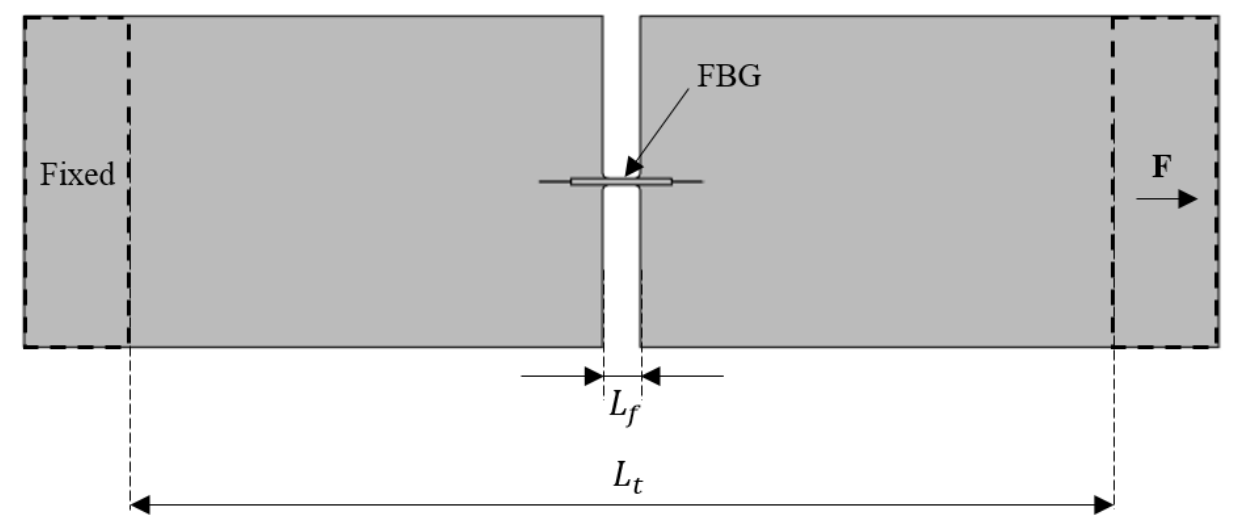

Figure 1. Top view of a transducer based on the design reported in [14] with outer dimensions of $380 \times 105 \mathrm{~mm}$.

For a transducer relying on a package composed of materials with different Young moduli such as reported in [14] and schematically depicted in figure 2, the amplification $A$ can be calclulated as

$$
A=\frac{1}{\alpha_{L}+\alpha_{E}-\alpha_{L} \cdot \alpha_{E}}
$$

where $\alpha_{E}=\left(E_{1} \cdot A_{1}\right) /\left(E_{2} \cdot A_{2}\right)$ and $\alpha_{L}=L_{1} / L$. The elastic moduli and cross-sectional areas of the portions with materials 1 and 2 are $E_{1}$ and $E_{2}$, and $A_{1}$ and $A_{2}$, respectively. One increases the strain measurement resolution of the system by ensuring that the elastic modulus of material 1 is much smaller than that of material $2\left(E_{1}<<E_{2}\right)$, such that

$$
A \approx \frac{1}{\alpha_{L}}=\frac{L}{L_{1}}
$$

For a total length of the sensor package of $600 \mathrm{~mm}$ and with the length of the FBG holding portion $L_{1}=120 \mathrm{~mm}$, one achieves at largest a strain amplification of 4.4. A key issue with this transducer is its manufacturing and the handling of an embedded tube in which a bare optical fiber equipped with an 
FBG is sleeved and fixed at two extremities. Furthermore, a transducer length of $600 \mathrm{~mm}$ is not always sufficiently small to allow for quasi-distributed measurements.

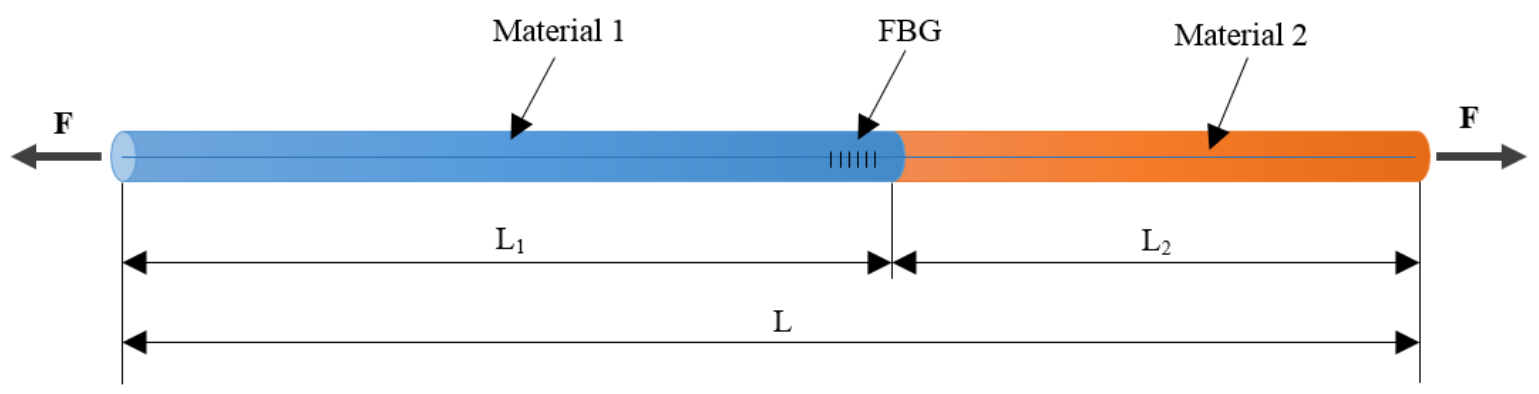

Figure 2. A transducer based on the design reported in [14] that uses two different materials with different stiffness's.

Another interesting device though has been developed by Paulo Jr et al [17] with the intention to apply a controlled strain to an FBG in order to tune the Bragg wavelength to a desired value by means of a piezoelectric (PZT) actuator. It was designed to magnify the displacement induced by the PZT actuator and hence not for strain measurements based on amplification of said strain. The device consists of two supports and a beam, with one support anchored and the other exposed to the input force generated by the PZT actuator. The principle of operation relies on a rotation about an axis located between two hinges, to obtain a so-called notch-type spring. This principle initially inspired the design of our strainamplifying transducer, which is detailed in the following Section.

\section{Design and numerical analysis of the strain-amplifying transducer}

Figure 3 shows the basis of the structural model of our strain-amplifying transducer. It features a symmetric double cantilever structure and contains two flexible interconnections between rigid portions. The pre-defined spacing between the two rigid portions allows for easy installation of an optical fiber holding a FBG. Two fixation points also enable bolting the transducer to a concrete structure. A displacement applied to the fixation points results in a relative displacement at the spacing in the central section that is larger than the relative displacement applied to the transducer itself. As already stated, the gauge length should be sufficiently small to allow for quasi-distributed measurements, whilst remaining sufficiently long to integrate the spatial strain variations inherent to the inhomogeneous nature of concrete. We adapted the size of the transducer shown here to enable such measurements on a lab-scale concrete beam. The dimensions are $380 \mathrm{~mm}$ by $105 \mathrm{~mm}$, with a gauge length $L_{T}$ of $275 \mathrm{~mm}$. The first eigenfrequency of this transducer was numerically simulated to be 244 $\mathrm{Hz}$, which is well above the typical resonance frequencies of civil structures such as long pre-stressed concrete bridges. This transducer therefore already meets several of the specifications outlined in Section 1. 


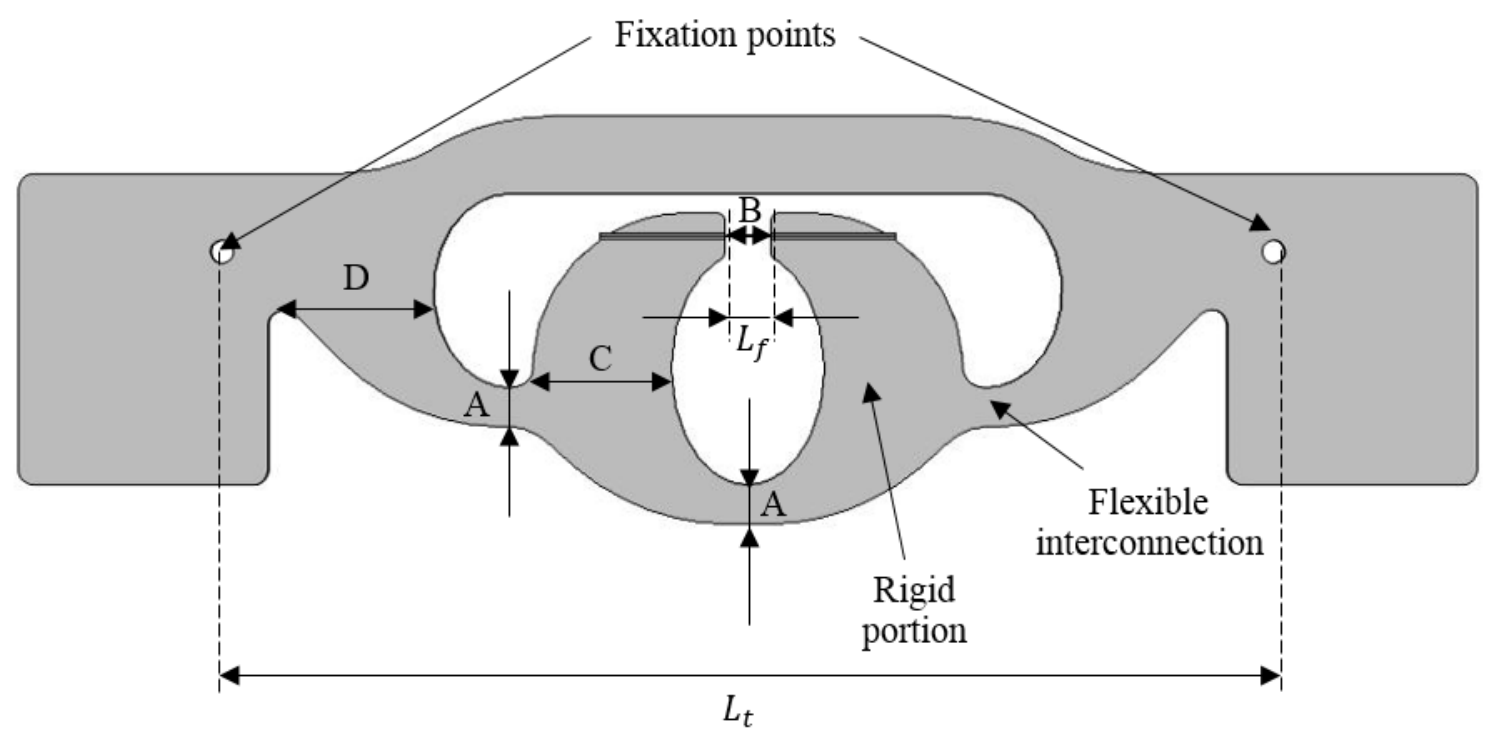

Figure 3. Transducer design with indication of the lengths $\mathrm{L}_{t}$ and $\mathrm{L}_{\mathrm{f}}$ that are used to define the strain amplification and the key dimensions A, B, C and D of the transducer which were optimized.

To mount the FBG, we glued fiber portions at both sides of the FBG using a two-component adhesive into v-grooves fabricated using a standard milling machine. In the design, the transducer consists of two mounting brackets which allow for calibration in a tensile machine.

To optimize the design of the transducer we have built a three-dimensional (3D) finite element (FE) model in COMSOL $®$ [18]. The model takes into account the material of the transducer, the glass optical fiber (the diameter of the cladding and the polymer coating of the fiber are $125 \mu \mathrm{m}$ and $195 \mu \mathrm{m}$, respectively) and the adhesive applied to fix the fiber in the v-grooves. The simulations also consider a reference fiber attached to the surface of the brackets which allows measuring the strain applied to the transducer itself (see figure 4). The mesh contains a total of 537638 elements (tetrahedral, pyramid and hexahedral). Refined meshes are also generated at the location of the optical fiber and the adhesive, featuring in total 382096 elements. The material parameters used in the 3D FE analysis are listed in table 2 . Note that the material for the transducer was chosen following simulations with different values of the Young Modulus corresponding to different materials. The achievable strain amplification of the transducer tends to converge when the Young modulus of the material of which it is made exceeds 50 GPa (figure 5). We eventually fabricated this transducer in aluminum owing to its relatively high strength-to-weight ratio, low cost and resistance to corrosion. 


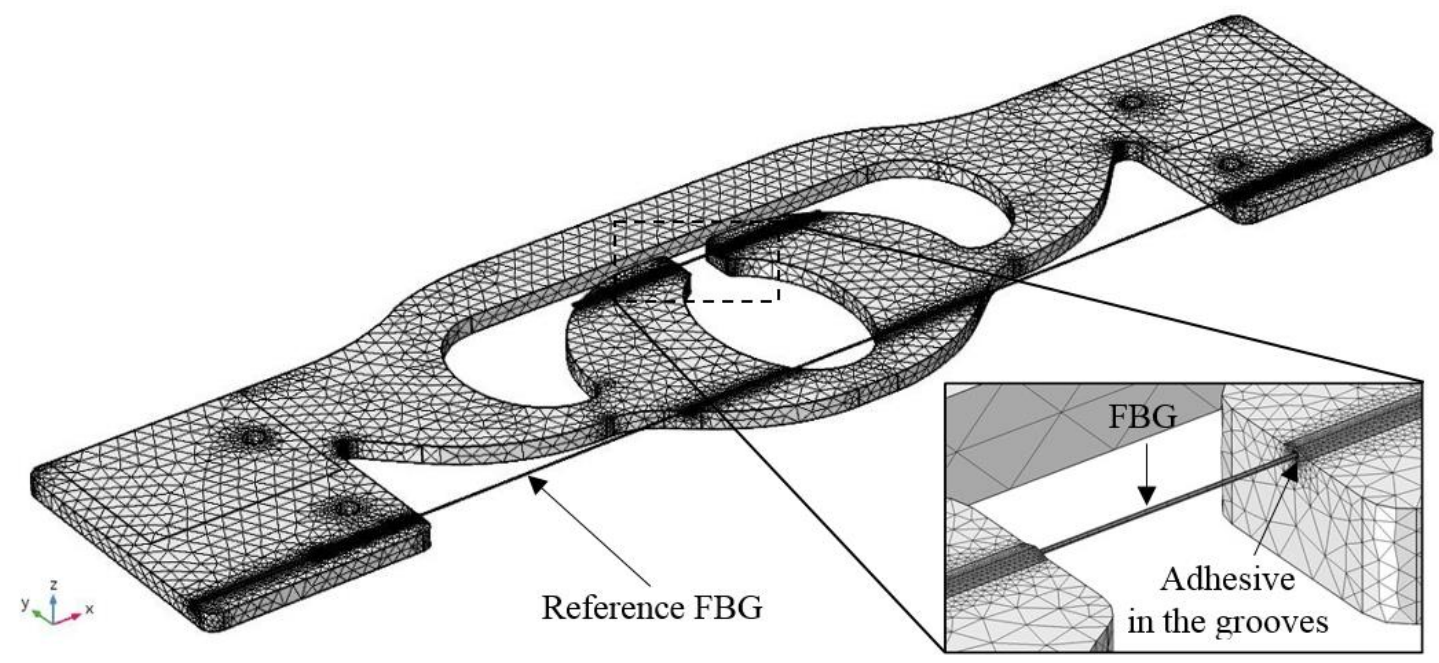

Figure 4. Illustration of the three-dimensional finite element model used for the analysis in COMSOL®.

Table 2. Material properties used in the simulations.

\begin{tabular}{lll}
\hline Component & Young Modulus $(\mathrm{GPa})$ & Poisson ratio (-) \\
\hline Silica fiber & 74 & 0.3 \\
Fiber coating & 1.5 & 0.3 \\
Adhesive & 13 & 0.35 \\
Aluminum & 70 & 0.33 \\
\hline
\end{tabular}

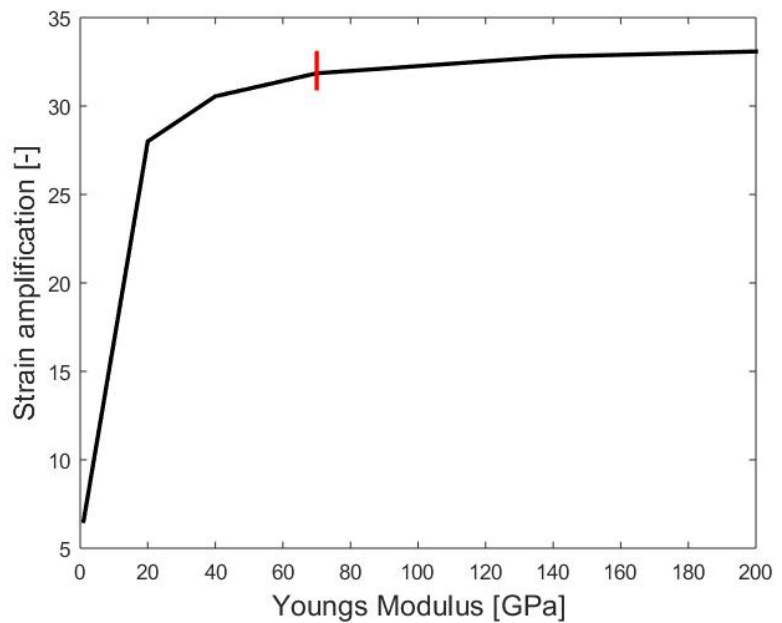

Figure 5. Dependence of strain amplification on the Young modulus of the transducer material. The red line indicates the strain amplification value obtained using the Young modulus of aluminum.

The boundary conditions in our simulations were defined as follows. One side of the transducer was fixed over an area covering most of the left mounting bracket to mimic the calibration setup shown in figure 8 and a tensile force of $100 \mathrm{~N}$ (indicated with $\mathbf{F}$ in figure 6) along the $\mathrm{x}$-direction was applied to the right mounting bracket. This value is equivalent to the load applied in the tensile test that will be discussed in Section 4. The resulting strain amplification $A$ was calculated by dividing the strain experienced by the FBG in the central section $L_{f}$ by the strain experienced by the reference FBG $L_{T}$ (see also equation (1) and figure 3). The total displacement throughout the transducer under load is illustrated by means of a grey-scale in figure 6 . A force applied to the mounting brackets results in a relative 
displacement at the spacing in the central section that is larger than the relative displacement applied to the transducer itself.

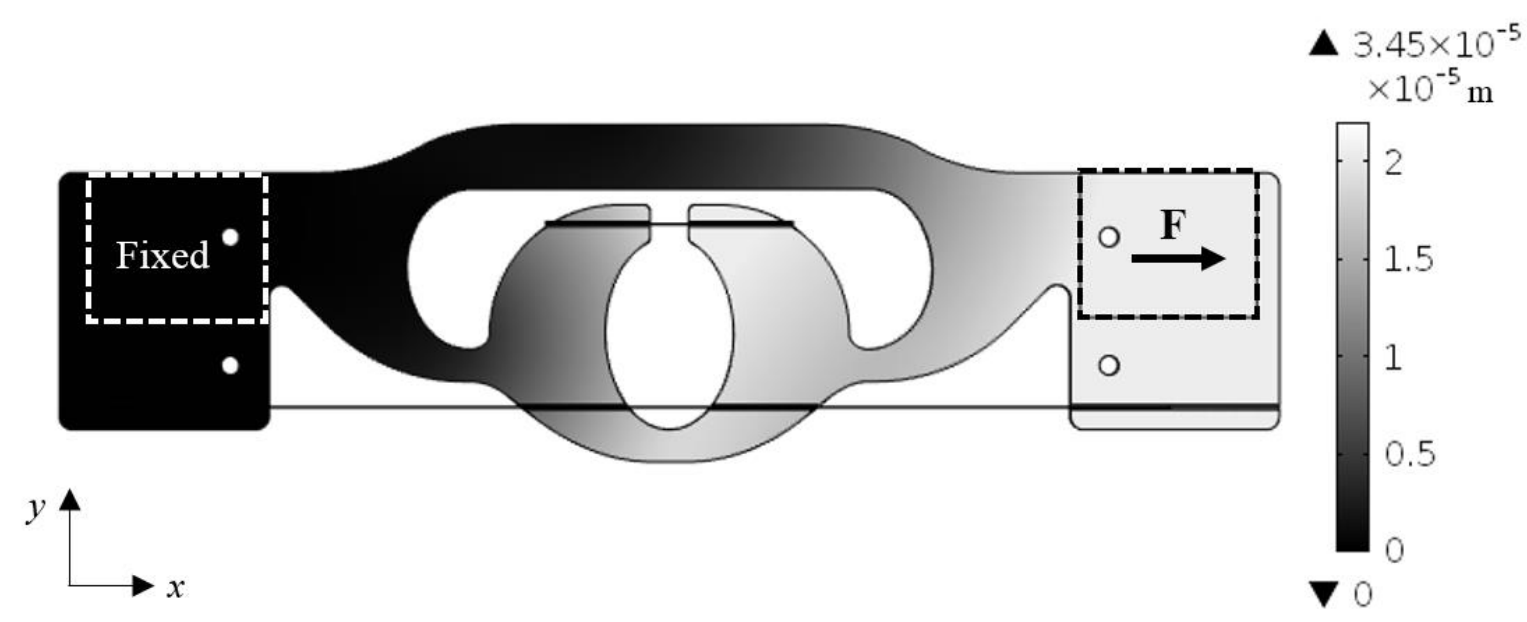

Figure 6. Simulated result for the total displacement across the strain-amplifying transducer when loaded with a tensile force of $100 \mathrm{~N}$ in the $+\mathrm{x}$ direction.

The key dimensions A, B, C and D of the transducer (see figure 3) were optimized using the COMSOL® FE model in order to achieve the highest strain amplification given the outer dimensions of $380 \times 105$ $\mathrm{mm}$ of the transducer. One of the criteria used for the optimisation was that flexible interconnections can not to be too narrow (below $7 \mathrm{~mm}$ ) to avoid in-plane and out-of-plane bending. Parameter B should not be smaller than $8 \mathrm{~mm}$ to allow for sufficient space for installing the FBG sensor. We optimized the key dimensions by varying their value in steps of $1 \mathrm{~mm}$ in the following ranges: 7-10 $\mathrm{mm}$ for A, 8-12 $\mathrm{mm}$ for $\mathrm{B}$ and in steps of $10 \mathrm{~mm}$ : $50-70 \mathrm{~mm}$ for $\mathrm{C}$ and $57-77 \mathrm{~mm}$ for $\mathrm{D}$. The value of strain amplification after optimization was 31.9.

We also considered the effect of the transducer's thickness in the range from $3 \mathrm{~mm}$ to $9 \mathrm{~mm}$ every 2 $\mathrm{mm}$. For each value we calculated the spring constant and the lowest eigenfrequency. To do so we fixed one side of the transducer whilst loading the other side along the $+\mathrm{x}$ direction with a total force of 100 $\mathrm{N}$. We used the elongation between the fixation points to calculate the value of the spring constant (table 3). Each of the simulated transducers with different thicknesses has a spring constant below $10^{7} \mathrm{~N} / \mathrm{m}$ and a first eigenfrequency above $50 \mathrm{~Hz}$. We eventually manufactured the transducers with a thickness of $5 \mathrm{~mm}$ as this provides the highest strain amplification.

Table 3. Summary of the transducer characteristics for different thicknesses.

\begin{tabular}{cccc}
\hline $\begin{array}{c}\text { Thickness } \\
(\mathrm{m})\end{array}$ & $\begin{array}{c}\text { Spring constant } \\
\left(10^{6} \mathrm{~N} / \mathrm{m}\right)\end{array}$ & $\begin{array}{c}\text { Strain amplification } \\
(-)\end{array}$ & $\begin{array}{c}\text { First eigenfrequency } \\
{[\mathrm{Hz}]}\end{array}$ \\
\hline 3 & 2.9 & 29.8 & 148 \\
5 & 4.6 & 31.9 & 244 \\
7 & 6.3 & 30.8 & 338 \\
9 & 8.0 & 31.2 & 409 \\
\hline
\end{tabular}

\section{Experimental details}

We have manufactured 4 transducers according to the design described in the previous Section using laser cutting. We fabricated the grooves with a standard milling machine. The depth and the width of the grooves are $0.3 \mathrm{~mm}$ and $1.5 \mathrm{~mm}$, respectively. For the FBG sensors we chose to use commercially available ORMOCER ${ }^{\circledR}$ coated Draw Tower Gratings (DTG®) [19], with a coating diameter of $195 \mu \mathrm{m}$. The length of the DTGs is $8 \mathrm{~mm}$ and the reflectivity is above $20 \%$. Before installation of the FBGs, we 
cleaned the grooves with isopropanol. We then equipped each transducer with two FBGs, glued in the grooves using X60 adhesive from HBM [20] (figure 7). To control the amount of adhesive we applied it by means of a syringe with needle. A first grating (DTG1) is installed near the fixation points on the mounting brackets in order to provide a reference measurement of the total strain applied to the transducer. A second grating (DTG2) is installed in the region with amplified strain. The red and green lines in figure 7 indicate the regions were the adhesive was applied in the corresponding grooves.

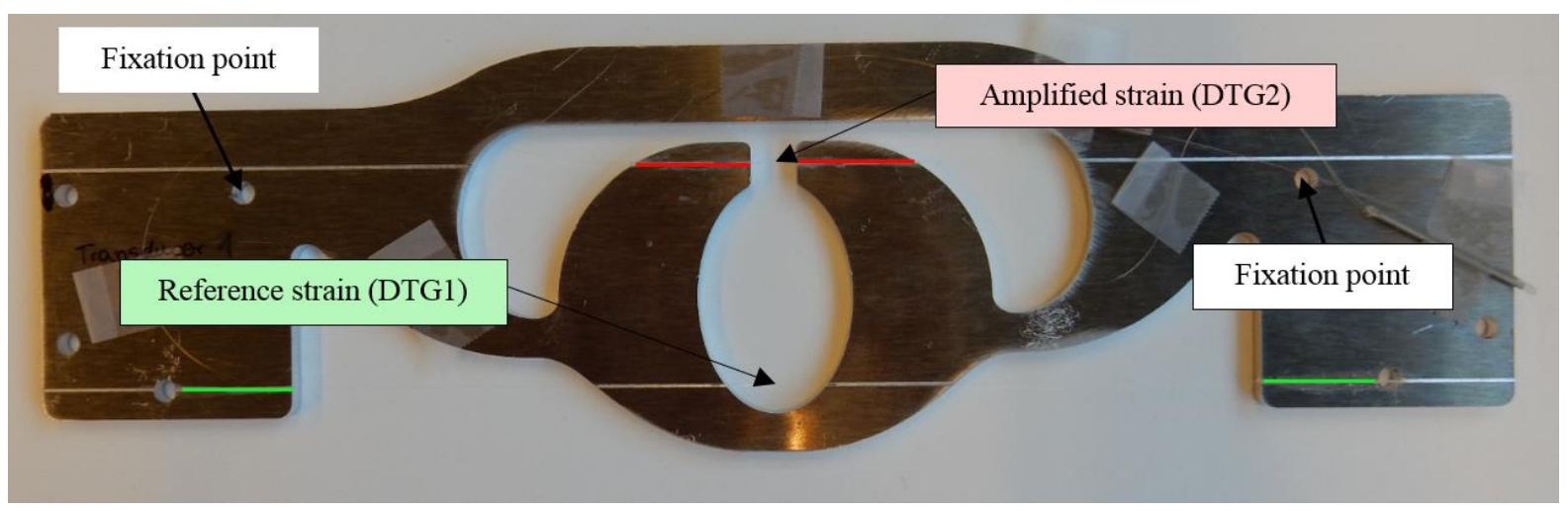

Figure 7. Photograph of the fabricated transducer with indication of the location of the FBGs and the glued sections.

To calibrate the transducers we carried out tensile tests on a Schenck Trebel test bench (figure 8). We increased the applied axial load from $0 \mathrm{~N}$ to $100 \mathrm{~N}$ at a constant displacement speed $(0.2 \mathrm{~mm} / \mathrm{min})$ and repeated the load/unload cycle three times. We characterized four transducers to check on the repeatability of the manufacturing and results.

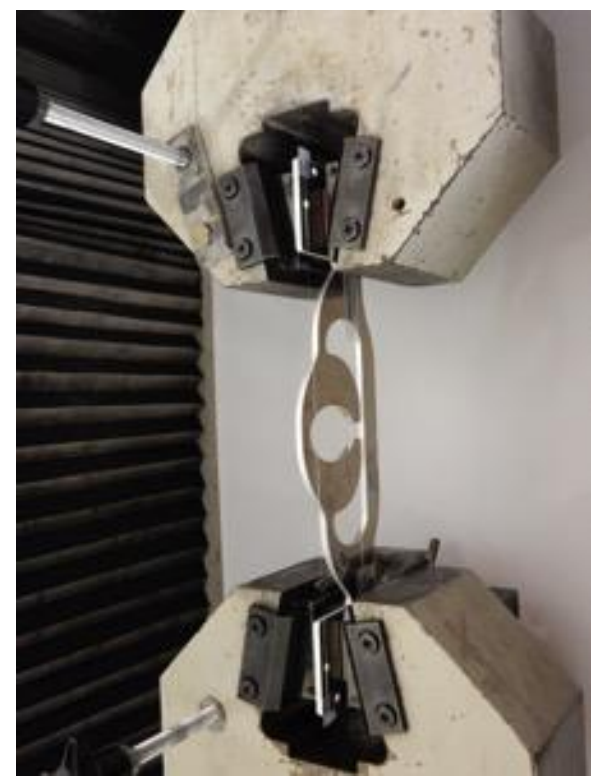

Figure 8. Transducer clamped in the tensile machine during tensile tests.

We monitored the reflection spectra and the shift of the Bragg wavelengths with a Micron Optics SM125-500 interrogator, featuring a Bragg wavelength measurement accuracy of $1 \mathrm{pm}$ at a measurement frequency of $2 \mathrm{~Hz}$. During the tests we also monitored the temperature using another FBG sensor. We found that the temperature was sufficiently stable $\left( \pm 0.15^{\circ} \mathrm{C}\right)$ to allow neglecting the influence of temperature variations.

Figure 9(a) shows the reflection spectrum of DTG1 and DTG2 in unloaded condition and when loaded with a $100 \mathrm{~N}$ tensile force (see also figure 8) for one of the transducers. Figure 9(b) shows the relation between the strain measured by both DTGs during the 3 load/unload cycles. To calculate the strain 
amplification, we performed a weighted linear fit of the recorded amplified strain versus the recorded reference strain. We have assigned weights in the fit that are proportional to the strain amplitude, since low amplitude strain results in a larger relative error than high amplitude strain. The slope of the linear fit of these data points provides the experimental value for the strain amplification $A$. We tested the four transducers, which yielded strain amplification factors of 33.3, 33.8, 37.7 and 36.7, with a Pearson Correlation Coefficient for the linear regressions of 0.996, 0.987, 0.991 and 0.996, respectively. The variation between the strain amplification factors essentially stems from small differences in the dimensions of the grooves and from differences in the amount of adhesive used to attach the sensors. Note that the strain amplification values of all the transducers are close to that obtained in the simulation (31.9).

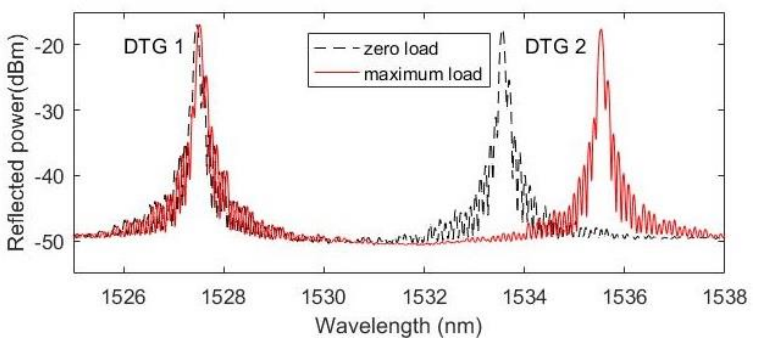

(a)

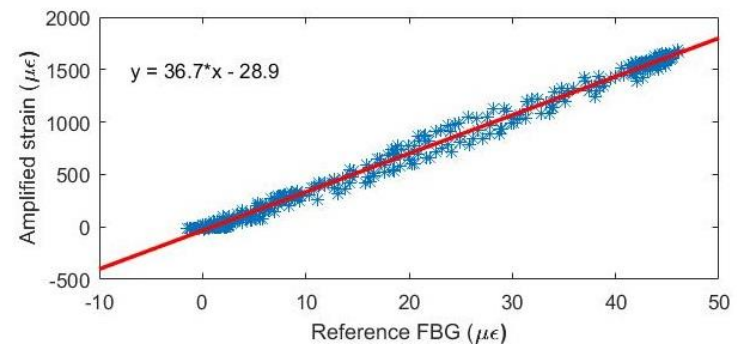

(b)

Figure 9. (a) Reflected power spectrum of the DTGs on one of the fourth strain-amplifying transducer under zero and maximum load. (b) The strain amplification is derived as the slope of the linear fit of amplified strain versus reference strain.

\section{Static and dynamic test on the concrete beam}

In order to evaluate the operation of our transducer in view of measuring the targeted small-level dynamic strain measurements on concrete structures, we have also conducted an experiment on a fullscale pre-stressed concrete beam (figure 10 (a)). The I-shaped beam had a length of $5.0 \mathrm{~m}$ and a variable height up to $0.9 \mathrm{~m}$. It rested on a supporting steel table by means of two supports located at $1.0 \mathrm{~m}$ from the extremities. For quasi-static loading, the boundary conditions approximated these of a simply supported beam. The four transducers were attached to the bottom of the concrete beam (figure 10 (b)) using dedicated mounting supports that were first glued to the surface of the concrete beam (see figure $10(\mathrm{c}))$. 
a)

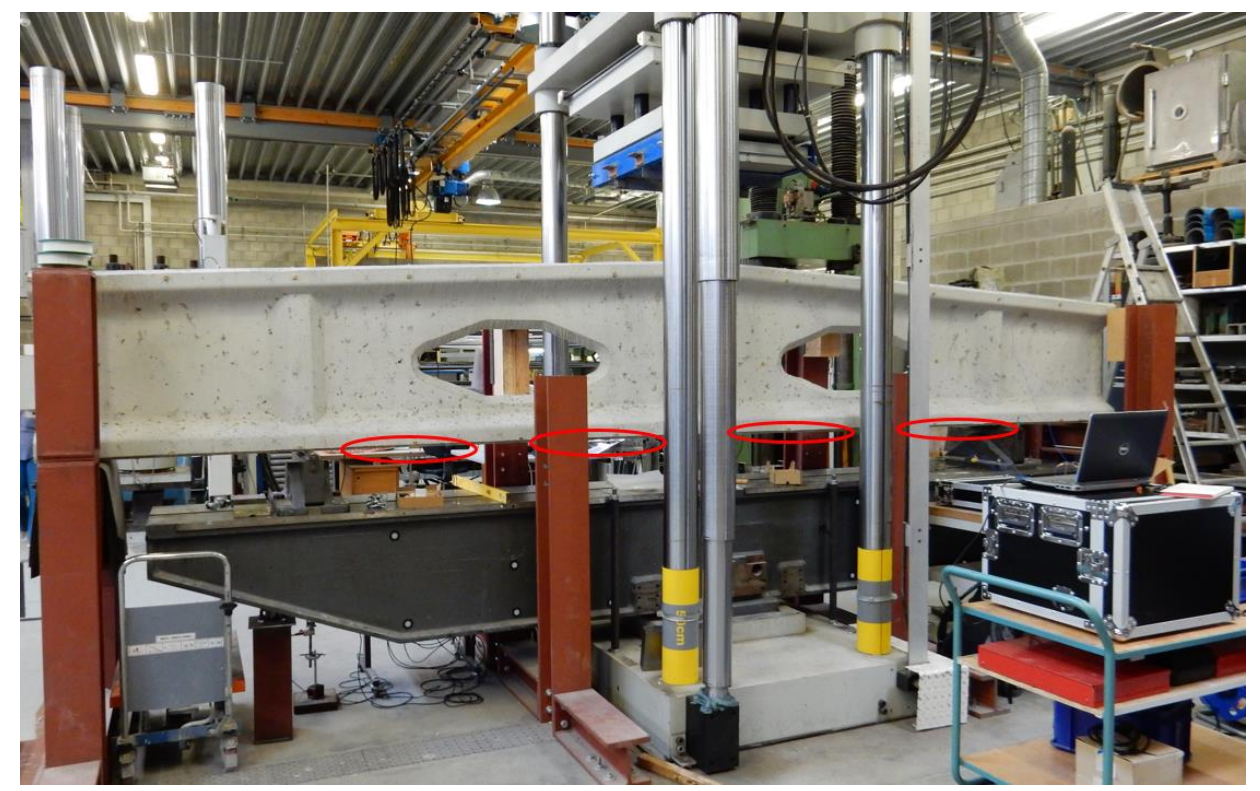

b)

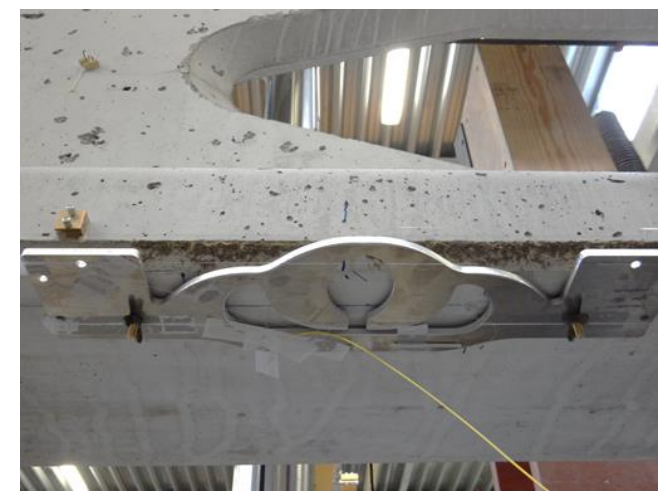

c)

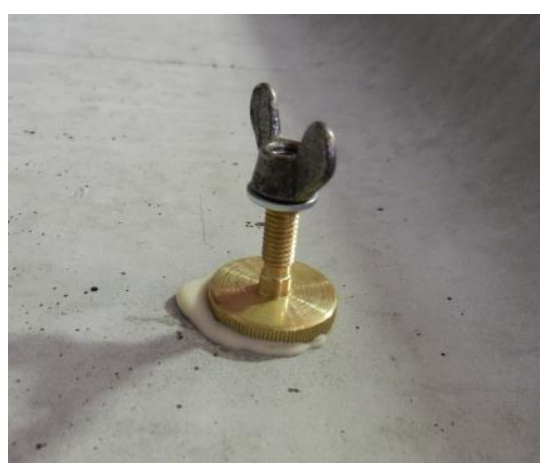

Figure 10. Photograph of the side of the pre-stressed concrete beam in the 3-point bending setup (red ellipses indicate places where transducers were installed) (a), showing one of the transducers fixed to the bottom of the beam (b) and dedicated mounting supports that were glued to the surface of the concrete beam (c).

Static and dynamic tests were performed in collaboration with the Building Materials and Building Technology Section of KU Leuven. In the static 3-point bending test, a vertical load was applied to the beam at one location in the middle of the beam. The maximum applied force was $100 \mathrm{kN}$. For the dynamic tests the beam was excited with a hammer during 30 seconds, such that the hammer impacted the structure from the top at one of its corners.

Figure 11(a) shows strain obtained with a selected transducer during one cycle of increasing and decreasing load during the static test. As expected the strain measured by DTG2 (blue line) located in the central section of the transducer is significantly larger than that recorded by the reference sensor DTG1 (red line). The minimum strain measured by DTG2 was $-420 \mu \varepsilon$, whilst that obtained from DTG1 was $-13 \mu \varepsilon$. It is also noticeable that the signal obtained on the reference FBG is much more noisy compare to the signal where the strain is amplified. The negative value indicates that the transducer experienced compressive strain. Figure 11 (b) shows the relation between the strain measured by both DTGs during the static test up to a maximum load of $100 \mathrm{kN}$. To calculate the strain amplification from the static test we used the same weighed linear fit as discussed in Section 4. The slope of the linear fit of data points for four transducers result in an experimentally obtained value for the strain amplification of 32.3, 30.3, 33.4 and 31.4, with a Pearson Correlation Coefficient for the linear regressions of $0.997,0.978,0.996$ and 0.978 , respectively. The experimental results and the numerical results (determined from the FE model) are in good agreement, with a relative error smaller than 5\%. This supports the validity of our FE model. 
a)

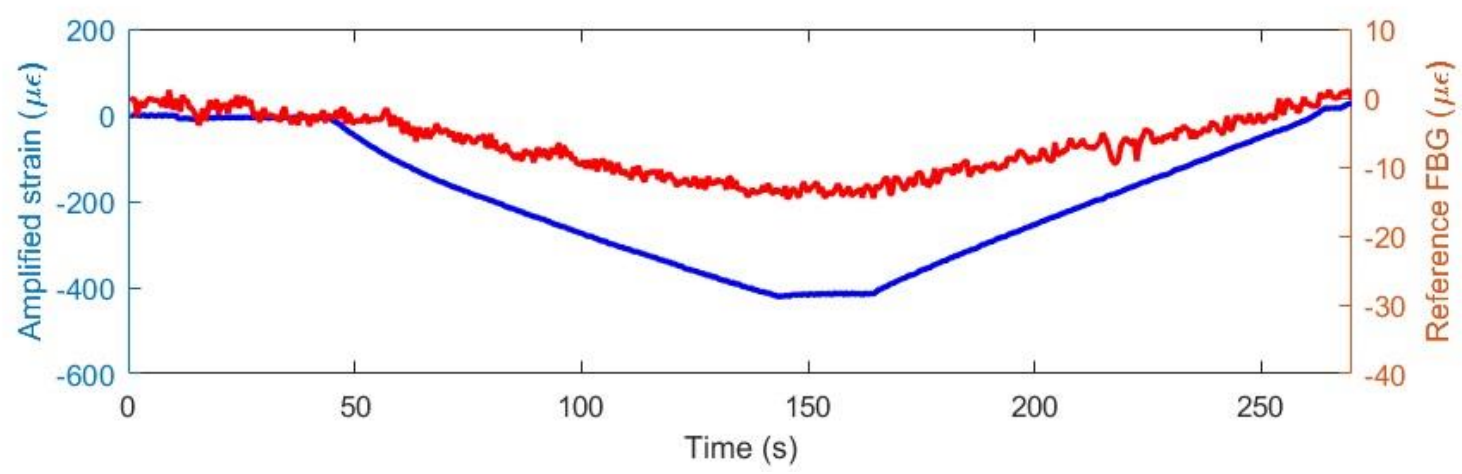

b)

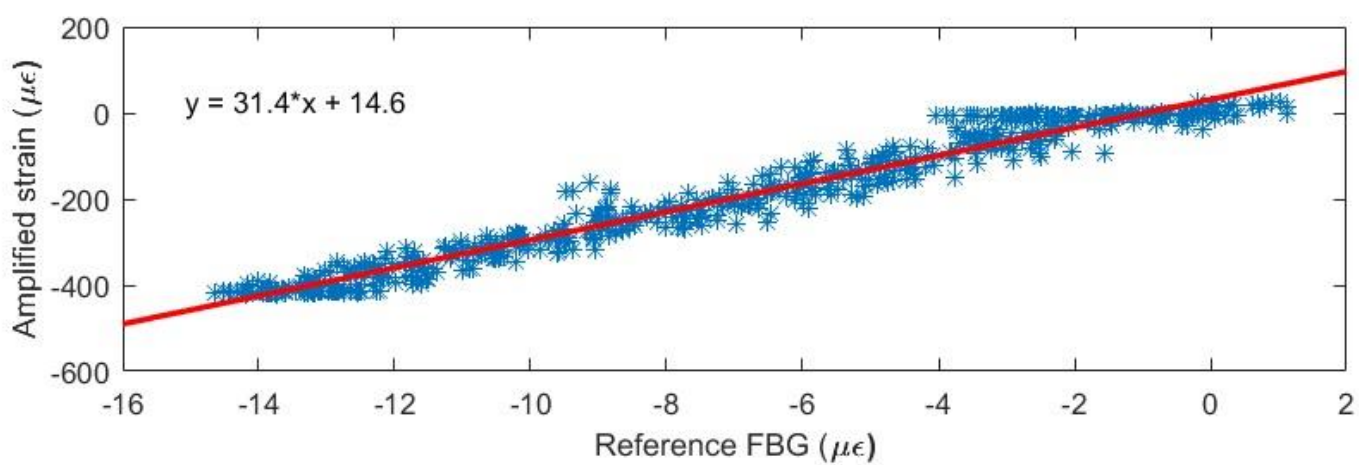

Figure 11. Strain measured on the reference FBG (DTG1) and on the FBG experiencing the amplified strain (DTG2) for the fourth transducer during the static 3-point bending test under maximum load $100 \mathrm{kN} \mathrm{(a),} \mathrm{relation} \mathrm{between} \mathrm{the} \mathrm{strain} \mathrm{measured}$ by both DTGs during the static test (b).

Figure 12 reports measurements obtained from the same selected transducer during the dynamic tests. To analyse the data we first applied a high pass filter to remove low frequency noise resulting from temperature and optical power fluctuations of the light source. The cut-off frequency of the high pass filter was $0.5 \mathrm{~Hz}$. The maximum strain during hammer impacts measured with DTG2 (amplified strain) is close to $140 \mu \varepsilon$, whilst for DTG1 (reference strain) the maximum strain was close to $4 \mu \varepsilon$ (figure 12(a)). To calculate the strain amplification from the dynamic test we again applied the same weighed linear fit. The strain amplification calculated from the slope of the linear fit of these data points gives a value of 36.9, 34.5 and 31.6, with a Pearson Correlation Coefficient for the linear regressions of 0.966, 0.927 and 0.906 for the transducer 2, 3, 4, respectively. For transducer 1 the signal from the reference FBG was not recorded, but the measured strain from the amplified strain FBG was close to $140 \mu \varepsilon$, i.e. similar to that obtained with the other transducers. The values of strain amplification calculated from the dynamic tests are higher comparing with the static tests what can be caused by a change in the boundary conditions during these two experiments. 
a)

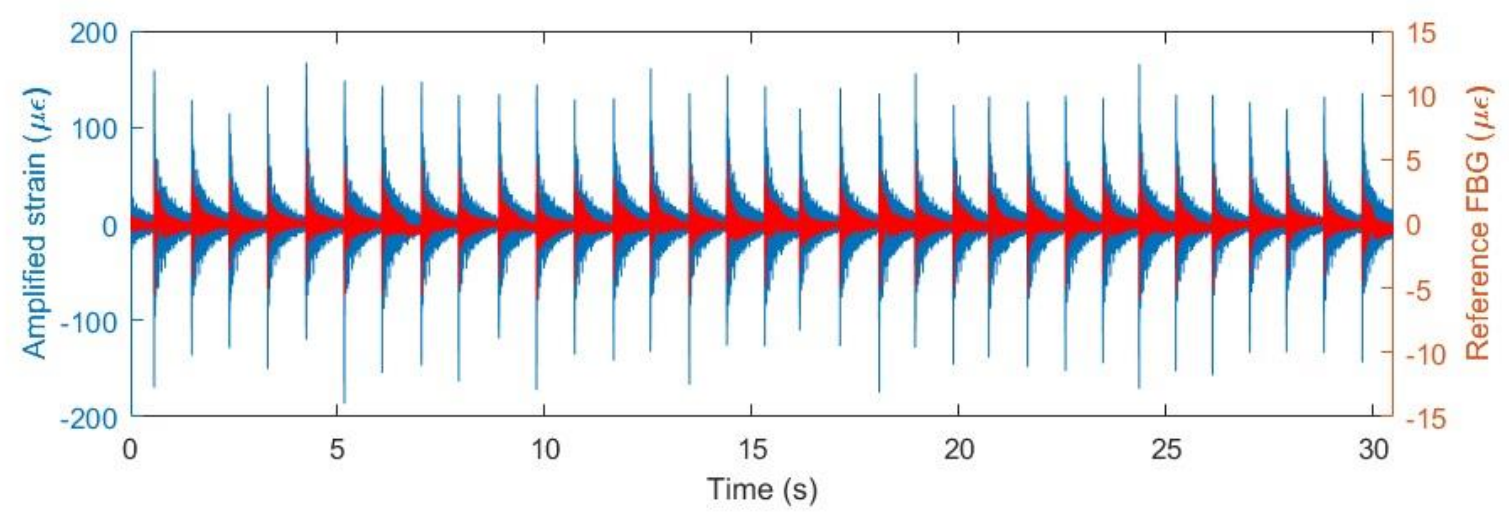

b)

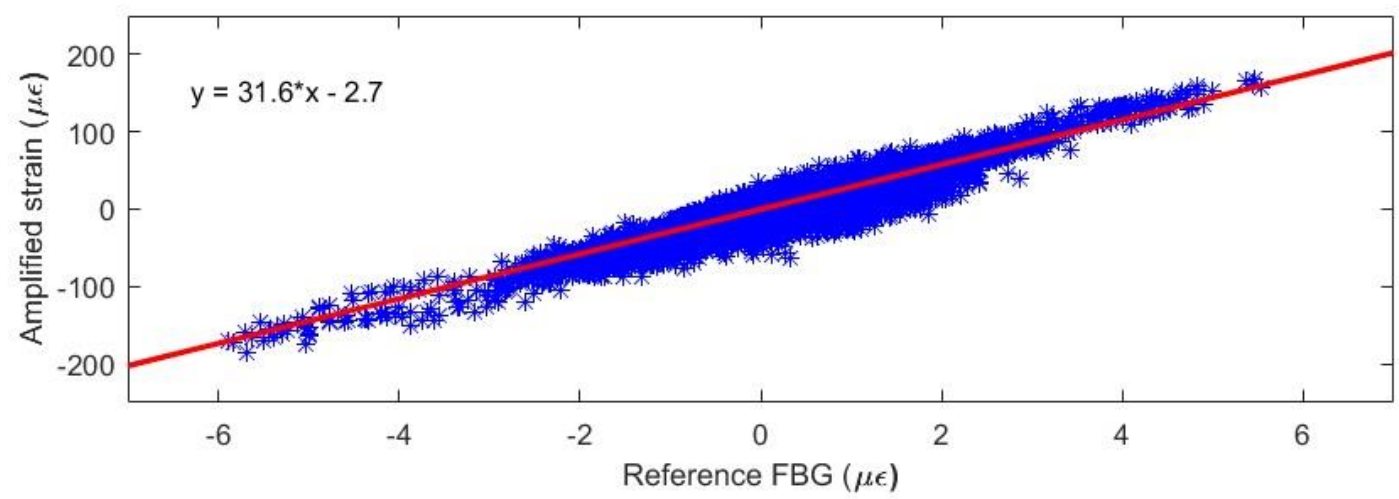

Figure 12. Strain measurements from reference FBG and amplifying strain FBG on the fourth transducer obtained during dynamic tests (a), relation between the strain measured by both DTGs during the dynamic test (b).

\section{Conclusions}

We proposed a novel strain amplifying transducer using fiber Bragg grating based sensors for dynamic strain measurements on concrete civil engineering structures. The basic idea is to utilize a symmetric cantilever structure to enlarge strain measured by the FBG sensor compared to the strain applied to the transducer itself. We have built a 3D FE model for determining the strain level on the FBG sensor installed in the central section of the transducer. The strain amplification obtained from the simulations equals 31.9. We have verified the operation of the transducer by means of tensile test experiments on four transducers and by static and dynamic tests on a concrete beam equipped with these transducers. The average experimentally derived strain amplification during the latter static experiments over the four transducers is 32 , which agrees very well with the finite element modelling. The variations between the strain amplification for calibration in the tensile machine, static and dynamic tests can by caused by different boundary conditions during experiments. Our transducer features an eigenfrequency of 244 $\mathrm{Hz}$, which is higher than the typical resonance frequencies of civil structures such as long pre-stressed concrete bridges. We designed the spring constant of the transducer to be below $10^{7} \mathrm{~N} / \mathrm{m}$ in order to minimally affect the behavior of the structure to which it is attached. Furthermore, to allow for simple installation and replacement of the transducer, we used dedicated mounting supports. Our transducers can be fabricated in large amounts and in a repeatable manner from laser-cut aluminum, and are therefore relatively inexpensive. All these features allow considering the use of this transducer for accurate dynamic strain monitoring of civil structures in operational conditions with potential for overcoming some of the challenges encountered in sensor systems for vibration-based damage identification. 


\section{Acknowledgements}

This research has been performed as part of project G099014N: "Robust vibration-based damage identification with a novel high-accuracy strain measurement system" funded by the Research Foundation - Flanders (FWO). T. Geernaert is post-doctoral research fellow of FWO. The tests that are discussed in section 5 were performed in collaboration with Kristof De Wilder and Lucie Vandewalle from the Building Materials and Building Technology Section of KU Leuven. The authors also would also like to acknowledge financial support from the Methusalem and Hercules Foundations.

\section{References}

[1] A. Deraemaeker and K. Worden, New Trends in Vibration Based Structural Health Monitoring, Vienna: Springer, 2010.

[2] J. Brownjohn, A. D. Stefano, Y.-L. Xu, H. Wenzel and A. Aktan, "Vibration-based monitoring of civil infrastructure: challenges and successes," J. Civil Struct. Health Monitoring, vol. 1, no. 3-4, pp. 79-95, 2011.

[3] A. Pandey, M. Biswas and M. Samman, "Damage detection from changes in curvature mode shapes," J. Sound Vib., vol. 145, no. 2, p. 321-332, 1991.

[4] E. Reynders, G. D. Roeck, P. Bakir and C. Sauvage, "Damage Identification on the Tilff Bridge by Vibration Monitoring Using Optical Fiber Strain Sensors," Journal of Engineering Mechanics, vol. 133, no. 2, pp. 185-193, 2007.

[5] F. d. Santos, B. Peeters, J.Lau, W. Desmet and L. Goes, "An overview of experimental strainbased modal analysis methods," in 26th International Conference on Noise and Vibration engineering, Proceedings of ISMA2014, Leuven, 2014.

[6] B. Gliši'c and D. Inaudi, Fibre optic methods for structural health monitoring, Chichester: John Wiley \& Sons, 2007.

[7] A. Othonos and K. Kalli, Fiber Bragg Gratings: Fundamentals and Applications in Telecommunications and Sensing, Artech House Print on Demand, 1999.

[8] Y. M. Gebremichael, "A field deployable, multiplexed Bragg grating sensor system used in an extensive highway bridge monitoring evaluation tests," IEEE Sens. J., vol. 5, no. 3, p. 510-519, 2005.

[9] T. H. T. Chan, L. Yu, H. Y. Tam, Y. Q. Ni, S. Y. Liu, W. H. Chung and L. K. Cheng, "Fiber bragg grating sensors for structural health monitoring of Tsing Ma bridge: background and experimental observation," Eng. Struct., vol. 28, no. 5, p. 648-659, 2006.

[10] L. Schulz, J. P. Conte and E. Udd, "Long-gage fiber optic Bragg grating strain sensors to monitor civil structures," Proceedings of SPIE, vol. 4330, p. 56-65, 2001.

[11] I. Kaundinya, E. Hamann and F. Heimbecher, "D200 Identification and Classification of European Bridge and Tunnel Types," SeRoN - Security of Road Transport Networks (FP7-ICTSEC-2007-1 Grant Agreement no.225354), Germany, 2011. 
[12] R. Fernández-Valderasa, N. Gutiérrez-Vázqueza and F. Lasagnia, "Monitoring of Strains in CFRP During Mechanical Testing Using Fiber Bragg Grating Sensors," in European Conference on Composite Material, Seville, 2014.

[13] J. He, Z. Zhou, H. Donga and G. Zhanga, "Study on a new kind of surface sticking strain sensor with sensitivity enhanced based on FBG," Proc. SPIE, vol. 6595, 2007.

[14] S. Li and Z. Wu, "Sensitivity Enhancement of Long-gage FBG Sensors for Macro-strain Measurements," Structural Health Monitoring, vol. 8, no. 6, pp. 415-423 , 2009.

[15] L. LI, D. Zhang, H. Liu, Y. Guo and F. Zhu, "Design of an Enhanced Sensitivity FBG Strain Sensor and Application in Highway Bridge Engineering," Photonic Sensors, Vol. 4, No. 2, 2014 , vol. 4, no. 2, pp. 162-167, 2014.

[16] Y. Tu and S.-T. Tu, "Fabrication and characterization of a metal-packaged regenerated fiber Bragg grating strain sensor for structural integrity monitoring of high-temperature components," Smart Materials and Structures, vol. 23, no. 3, 2014.

[17] P. Jr, C. Bavastri, J. Pereira, R. Oliveira, A. Pohl, D. Boni and M. Luersen, "Fiber Bragg grating tuning with notch-type spring device," Measurement Science and Technology, vol. 22, no. 8, p. $10,2011$.

[18] “COMSOL,” [Online]. Available: https://www.comsol.com. [Accessed 0102 2017].

[19] "FBGS,” [Online]. Available: https://www.fbgs.com. [Accessed 0102 2017].

[20] “"HBM",” [Online]. Available: https://www.hbm.com. [Accessed 0102 2017]. 